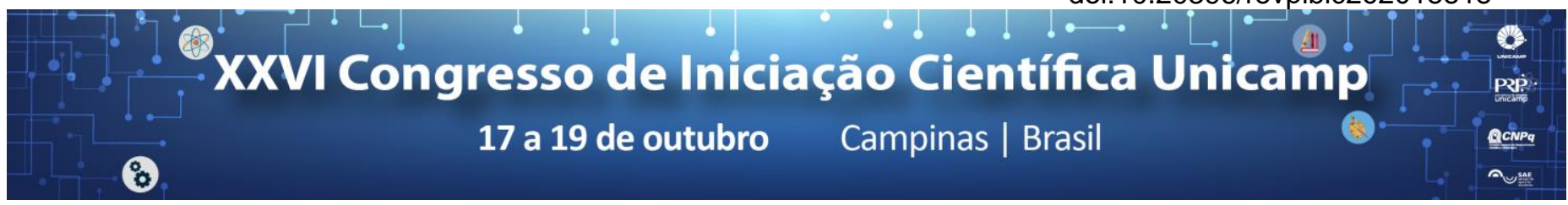

\title{
Production and phisical-chemical analysis of cationics liposomes and STEALTH® types, obtained from microfluidic tecnology for gene therapy application
}

\section{Leonardo Jose Montebugnoli ${ }^{\star}$ Lucimara Gaziola de la Torre, Ismail Eş}

\begin{abstract}
The research was focused on the production of lipid based vectors for gene therapy by microfluidic devices, particularly carionic liposomes (CL), and their complexation with plasmidic DNA (pDNA). Two types of microfluidic devices were proposed to achieve the goals, a diffusion based microfluidic device (D-MD) and a caotic advection based microfluidic device (CA-MD). The research showed that the CA-MD has a better productivity of CL while maintaining good properties. In the complexation studies, the D-MD was capable of make the complexes of genetic materials and liposomes (Lipoplexes).
\end{abstract}

\section{Key words:}

Microfluidic, Liposomes, Gene Therapy

\section{Introduction}

Gene therapy is the delivery of a genetic material (DNA or RNA) to a specific cell to achieve a therapeutical response. Genetic or non-genetic diseases are possible to be treated by this technique ${ }^{1}$. However, the immune system servers as a great barrier for the genetic material to surpass and enter the cell. Therefore, vectors were proposed with the function to protect and delivery the genes to the targets cells. The research was based on the production of CL, a non-viral vector. The incorporation of polyethylene glycol (PEG) has a great impact on the use of $C L$ in gene therapy because it provides the reduction of the size of the vesicle, stabilization of the particles and the better formation of the lipid bilayer due to a steric barrier, give an inert aspect to the particle and accumulate in cancerous tissues by the enhanced permeability and retention effect. CL with PEG are named STEALTH®.

Microfluidics emerges as a prominent field that enables the manufacturing several kinds of non-viral vectors with a simpler process and better control of the system. CL production in microfluidics by a D-MD (Image 1-A) was explored by Balbino, et al. $(2013)^{2}$. Two new devices (Image 1-B and C) CA-MD were investigated, in these pools were introduced along the channel to provide turbulence (Image 1-D). Because of the cationic characteristic of the phospholipids when in presence of genetic material, negatively charged, a complex is formed.

\section{Results and Discussion}

The production of CL (EPC, DOTAP and DOPE) was initially carried out using D-MD and produced samples were analyzed with Dynamic Light Scattering (Zetasizer Nano Malvern). D-MD produced CL with acceptable properties for transfection, which can be seen in Image 2. However, when PEG was introduced into the system precipitation occurred, it may be caused by the electrostatic interaction with the glass of the device, as well as the low solubility of the PEG in ethanol.

The CA-MD with 3 entries (Image 1-C) was proposed to solve this problem. It provided CL with properties as good as the D-MD-produced ones and succeed in the production of STEALTH CL. Then a simpler version of the CA-MD with just two inlets was proposed, which also proved to be effective. In the complexation studies it was considered the molar ratio of genetic material and the positively charged lipid as 3 so the amount of both the materials that need to

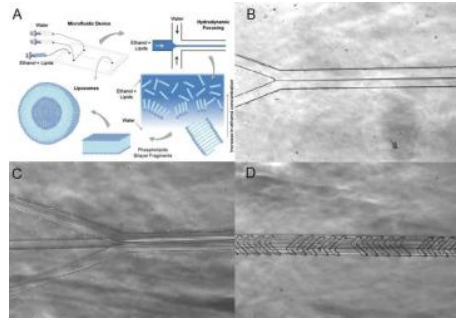

Image 1. A- D-MD'; B- CA-MD 2 entries; C- CA-MD 3 entries; $D$ - Herringbone pattern.

enter the channel was calculated. In the CA-MD (2 and 3 inlets) the complexation caused great precipitation in the channel, resulting in an impracticable process. It may be due to the advection which increases the impact of particles forming gigantic complexes. Although the D-MD succeed in making the complexes, probably because of the barrier offered from the water in the middle flow, which differs from the setup to produce the CLs in Image 1-A, offering a slow mass diffusion of both particles.

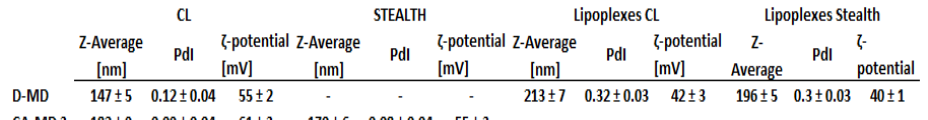

$$
\begin{aligned}
& \begin{array}{lllllll}
\text { CA-MD } 3 & 182 \pm 9 & 0.09 \pm 0.04 & 61 \pm 3 & 170 \pm 6 & 0.08 \pm 0.04 & 55 \pm 3
\end{array} \\
& \begin{array}{lllllll}
\text { CA-MD } 2 & 162 \pm 5 & 0.12 \pm 0.03 & 66 \pm 6 & 164 \pm 7 & 0.09 \pm 0.05 & 66 \pm 2
\end{array}
\end{aligned}
$$

\section{Image 2. Experimental results}

\section{Conclusions}

CA-MD with proved to be the best, of the studied devices, to produce CLs and STEALTHß in terms of productivity and simplest design (requiring less equipment). Meanwhile the D-MD was successful in the complexation of genetic material and the liposomes, however more studies to optimize and achieve greater properties for the lipoplexes.

\section{Acknowledgement}

This project could not be achieved without the aid of PIBIC/CNPq, the professor Lucimara Gaziola de La Torre as advisor, the doctorate student Ismail Eş and the LMF of the LNNano-CNPEM.

\footnotetext{
${ }^{1}$ Gardlik, R. et al.; "Vectors and delivery systems in gene therapy". Med Sci Monit, 2005, 11, n. 4,110-121.

2 Balbino, T. A. et al.; "Continuous flow production of cationic liposomes at high lipid concentration in microfluidic devices for gene delivery applications". Chemical Engineering Journal, 2013, 226, 423-433.
} 